\title{
How do patients spend their time in stroke rehabilitation units in England? The REVIHR study
}

\author{
N. CHOULIARA ET AL.
}

Patient activity in stroke rehabilitation units

[AQ0]

\author{
Niki Chouliara ${ }^{a}$ Rebecca Fisher ${ }^{\text {a }}$ Brian Crosbie $^{\text {a }}$ Boliang Guo $^{\text {b }}$ Nikola Sprigg $[$ [AQ7] DMarion \\ Walker [AQ1] \\ a. Division of Rehabilitation and Ageing, ool of Medicine, University of Nottingham, Nottingham, UK; \\ $b$. Division of Psychiatry and Applied Psy $\equiv$ gyy, School of Medicine, University of Nottingham, Nottingha, UK; \\ c. Division of Clinical Neuroscience, School of Medicine, University of Nottingham, Nottingham, UK
}

CONTACT Rebecca Fisher rebecca.fisher@nottingham.ac.uk Division of Rehabilitation and Ageing $\equiv$ ool of Medicine, University of Nottingham, Nottingham, NG7 2UH, UK

Received: 2019-07-31

Revised: 2019-11-21

Accepted: 2019-11-22

\section{ABSTRACT}

\begin{abstract}
Aim: To examine how patients spend their time in stroke rehabilitation units in England.Methods: We recruited 144 patients within a month after stroke from four stroke rehabilitation units and observed their activity type, interactions and location. Each participant was observed for 1 min every 10-minutes, for a total of $20 \mathrm{~h}$, over three consecutive days. Multilevel modelling was performed to assess differences across sites. Results: Across the four sites a total of 12,248 observations were performed. Patients spent on average $37 \%$ of the observed time inactive and $60 \%$ alone. A health care professional was present for $18 \%$ of the observations and patients' most frequent contact was with family members (19\%). Patients were mainly physically active in the presence of therapists, but they practiced self-care activities of daily living most frequently in the presence of nursing staff. There were limited opportunities for activity away from the bedside. Significant differences were found between the units, including patients' level of contact with rehabilitation assistants and nursing staff, but not in their time with occupational therapists and physiotherapists.Conclusions: Stroke patients in England spend a large proportion of their day inactive and alone. Opportunities to promote a rehabilitation focused environment may include: a) enhancing the role of rehabilitation assistants, b) supporting nursing staff in maximising opportunities for the practice of activities of daily living and c) involving family members in the rehabilitation process.
\end{abstract}

Keywords: Stroke ; rehabilitation units ; evidence-based practice ; behavioural mapping ; activities of daily living

\section{FUNDING}

National Institute for Health Research (NIHR) Collaboration for Leadership in Applied Health Research and Care East Midlands (NIHR CLAHRC EM)This work was supported by National Institute for Health Research (NIHR) Collaboration for Leadership in Applied Health Research and Care East Midlands (NIHR CLAHRC EM).[AQ2]

\section{Introduction}

Research evidence suggests that high intensity, specialist rehabilitation delivered in the context of an organised stroke unit can help reduce mortality and disability levels and improve long term outcomes [1-3]. Key elements of effective stroke rehabilitation have been identified and include the provision of early rehabilitation, once patients are medically 
able to participate, by a coordinated multidisciplinary team of specialists [4]. Rehabilitation is seen as an adaptive process, requiring practice and repetition of meaningful tasks to maximise recovery potential [2]. Cumulative evidence supports a dose response relationship in rehabilitation with more time spent in therapy leading to better functional outcomes [5]. To maintain and maximise the skills gained in therapy the importance of a stimulating environment that promotes patient activity and the practice of functional tasks has been highlighted in the literature [2,6].

Stroke clinical guidelines have been issued in many countries to facilitate the implementation of evidence based stroke rehabilitation in hospital [2,7-9]. However, studies in Europe, Australia and Canada suggest that evidencebased recommendations are not always met and national stroke audit findings from the United Kingdom highlight great variation in the quality of service provision [10-13]. There is a recognised need to better understand the delivery of stroke rehabilitation interventions in clinical settings to effectively address evidence-practice gaps [4,14].

Behavioural mapping is an established observational method which has been successfully used to assess the delivery of stroke rehabilitation [10,11]. It offers a more nuanced understanding of practice, necessary to ascertain those elements of care provision that should be enhanced and guide the development of targeted improvement strategies where they are most needed [15]. Previous behavioural mapping studies suggested that patients in England receive less rehabilitation than elsewhere in Europe and spend most of their time in rehabilitation units inactive and alone [16]. The introduction of a stroke improvement agenda in England (National Stroke Strategy and the Sentinel Stroke National Audit Programme) [12,13] has prompted developments in rehabilitation care but it is unclear whether these initiatives have translated into meaningful improvements in the rehabilitation experience of hospitalised stoke survivors.

The purpose of this behavioural mapping study was to examine the delivery of evidence-based rehabilitation by observing how patients spend their time in hospital stroke units in England. To date, behavioural mapping studies evaluating patients' use of time in stroke units have mainly focused on the degree of motor activity involved [10,11]. The assessment and practice of Activities of Daily Living (ADL) has received little attention in the literature despite being a key therapeutic target and a measure of success in stroke rehabilitation [17]. Guidelines recommend that stroke rehabilitation units encourage ADL practice to help patients regain their skills, maintain their dignity and prepare to return home [2]. It was therefore important to capture whether ADL practice is integrated in patients' daily routines in hospital rehabilitation settings.

Clinical guidelines view rehabilitation as a pervasive activity requiring the involvement of all multidisciplinary team staff members, family and caregivers in supporting the practice of functional skills gained in therapy and providing an active rehabilitation environment [2]. In view of this evidence, it was also important to investigate whether patients had adequate opportunities to interact with others and spend time away from their bedside. In summary, the study's objectives were to:

1. Examine what activities patients were engaged in during their stay in rehabilitation units, with particular interest in capturing ADL task practice.

2. Assess how much time patients spent alone or interacting with others and who was present during patient activity.

3. Examine what proportion of their day patients spent in different locations within the rehabilitation units.

4. Assess differences in rehabilitation provision between participating units.

\section{Methods}

\section{Participants and settings}

The study was conducted in four stroke rehabilitation units in the East Midlands region and was approved by the Nottingham 1 Ethics Committee (14/EM/1129). We followed a stratified purposeful sampling approach to capture variation in relation to: a) the size of the units and b) their geographical location (i.e. urban vs rural) and c) their performance in the Sentinel Stroke National Audit Programme - SSNAP audit at the time of recruitment (Table 1). Participants were recruited if they met the following criteria: a) confirmed first or recurrent stroke (as defined by the World Health Organisation) [18], b) first day of observations being within a month from stroke onset. Patients who 
received palliative care and those who were under 16 years of age were excluded from the study. Recruiting participants from stroke rehabilitation units ensured that the main purpose of their hospitalisation was rehabilitation [11].

Table 1. Stroke rehabilitation unit characteristics and staffing levels in relation to national standards (Sentinel Stroke National Audit Programme-SSNAP).

\begin{tabular}{|l|c|c|c|c|c|c|}
\hline & National results Median (IQR) & $\mathrm{A}$ \\
\hline Organisational SSNAP score* & NA & $\mathrm{A}$ & $\mathrm{C}$ & $\mathrm{D}$ \\
\hline No of beds & NA & $\mathrm{D}$ & $\mathrm{B}$ \\
\hline Location & NA & 21 & 15 & 24 & 27 \\
\hline Staffing Levels (WTE & \multicolumn{1}{|c|}{ Urban } & Urban & Rural & Rural \\
\hline Physiotherapy 10 beds) & $1.3(1.1,1.6)$ & 3.2 & 0.9 & 1.4 & 1.3 \\
\hline Occupational Therapy & $1.1(0.8,1.5)$ & 2.7 & 1.5 & 1.4 & 1 \\
\hline Speech \& Language Therapy & $0.5(0.3,0.8)$ & 1.1 & 0.4 & 1.2 & 0.7 \\
\hline Occupational & & & & & \\
\hline therapy assistants & $0.4(0.2,0.6)$ & 0.9 & 0 & 0.6 & 0.5 \\
\hline Physiotherapy & & & & & \\
\hline Rehabilitation assistants & $0.5(0.3,0.7)$ & 1.2 & 0 & 0.6 & 0.7 \\
\hline Nurses \& Healthcare Assistants & $3.2(2.9,3.6)$ & 3.9 & 2.5 & 3.3 & 3.0 \\
\hline
\end{tabular}

${ }^{*}$ Stoke care provision rated A (the highest) to E (the lowest) where A: first class service; B: good or excellent in many aspects; C: reasonable overall - some areas require improvement; D: several areas require improvement; E: substantial improvement required.

†WTE: whole time equivalent.

\section{Study design}

Observations in each site were conducted on 5 separate occasions over a 14-month period between January 2015 and March 2016. On each site visit, a sample of 10 patients who met the inclusion criteria were recruited and two research fellows observed a maximum of 5 participants each. In line with previous studies it was decided that this sample size would offer the best balance between data accuracy and practical constraints in daily healthcare practice [10]. Eligible participants were identified by members of the clinical team. If more than 10 patients fitted the eligibility criteria then a random sample of 10 patients was identified using a table of random numbers. Observations were conducted over a period of three days with a total of 15 days of observations per site. For two of the three days, observations took place from 8 AM until 5 PM. On one of the three days, observations were conducted between 3 PM and 8 PM. On each day researchers had three $10 \mathrm{~min}$ breaks and a $30 \mathrm{~min}$ lunch break. To avoid bias due to day to day fluctuations in clinical practice, observations in each site covered all $7 \mathrm{~d}$. To obtain a longer-term perspective of clinical practice in each setting, data collection alternated between the four sites. The same researchers collected data across sites to achieve consistency of observational method.

\section{Observational technique}

Using a behavioural mapping approach, each participant was observed every 10 min for 1-min duration, and the highest level of activity along with their location and people present were recorded using a list of pre-specified categories on a computer-based electronic schedule. Location of activity comprised eight possible categories (e.g. bedside, gym etc.) and people present during activity included 12 possible categories (e.g. alone, medical staff etc.). There were 27 possible activity categories with the focus being on ADL (e.g. transfers with help/independent). ADL categories were defined based on the Barthel Index of functional independence and included both self-care and mobility activities [19]. Patients were not followed off the ward nor intruded on if behind closed curtains or in private situations (e.g. visiting the bathroom). Once the patients were again visible, members of staff/carers were asked with 
a standard format to determine the activity in the unobserved period. The schedule items were developed in collaboration with clinicians and researchers and the comprehensiveness of the schedule and reliability of the coding process were piloted on a sample of four patients who were not included in the analyses. There was excellent inter-coder agreement between the two observers $(\kappa=0.93,<0.001)$.

\section{Patient casemix}

To characterise our population, the following information was extracted from patients' medical records: a) stroke syndrome (Oxford Community Stroke Project Classification) [20], b) severity of stroke at admission (determined with the National Institutes of Health Stroke Scale; NIHSS) [21], c) disability prior to the current stroke event as measured at admission (modified Rankin Scale; mRS) [22]. To determine participants' disability level at the time of observation we asked members of staff caring for our participants to complete the Barthel Index where a recent score was not available. The National Institutes of Health Stroke Scale -NIHSS scores were used to categorise patients as having mild (NHSS $<8$ ), moderate (NIHSS 8-16) or severe (NIHSS $>16$ ) stroke. Cog-4 [23], as a marker of cognitive performance, was also extracted from a subset of patients' most recent NIHSS.

\section{Data analyses}

Patient demographic and background information were summarised by hospital with mean (SD) for normally distributed variables, median (IQR) for skewed variables, frequency (percentage) for categorical variables. Multilevel modelling was performed to compare among hospitals with patient as higher level unit [24]. For categorical "location" and "activity" measures, multilevel multinomial logistic regression was conducted; for binary "people present" measure, multilevel logistic regression modelling was performed. STATA14 and MLwiN (2.36) were used for data analysis [25].

\section{Results}

\section{Unit and patient characteristics}

For confidentiality reasons the stroke units were anonymised and will be referred to as A, B, C and D. Their characteristics are shown in Table 1, where staffing levels are presented in relation to national standards at the time of observation as reported in the Sentinel Stroke National Audit Programme- SSNAP.

Across the four sites a total of 12248 observations were performed. All patients meeting inclusion criteria $(n=177)$ were approached and $144(82 \%)$ agreed to participate in the study. Six patients completed only $2 \mathrm{~d}$ of observations as they were discharged early or transferred to a different ward but were included in the analyses. The median number of days post-stroke on the first day of observation was 20 (IQR 10-30). No significant differences were found between the four units in terms of patient characteristics (Table 2).

Table 2. Patient characteristics across the four units.

\begin{tabular}{|l|r|r|r|r|}
\hline$N=144$ & $\mathrm{~A}(n=33)$ & $\mathrm{B}(n=35)$ & $\mathrm{C}(=39)$ & $\mathrm{D}(n=37)$ \\
\hline Age & & & & \\
\hline Median (IQR) & $73(63,85)$ & $75(64,84)$ & $76(72,83)$ & $80(73,84)$ \\
\hline Men & & & & \\
\hline$n(\%)$ & $19(57.6)$ & $17(48.6)$ & $18(46.1)$ & $19(51.3)$ \\
\hline NIHSS at admission & & & & \\
\hline Mean (SD) & $10.15(26.8)$ & $12.06(6.9)$ & $11.6(7.2)$ & $11.7(6.2)$ \\
\hline Barthel Index at time of observation & & & & \\
\hline $\begin{array}{l}\text { Median (IQR) } \\
\text { Side of symptoms }\end{array}$ & $32.5(20,52.5)$ & $25(15,40)$ & $40(5,60)$ & $15(10,42.5)$ \\
\hline
\end{tabular}




\begin{tabular}{|l|r|r|r|r|}
\hline$N=144$ & $\mathrm{~A}(n=33)$ & $\mathrm{B}(n=35)$ & $\mathrm{C}(=39)$ & $\mathrm{D}(n=37)$ \\
\hline Right & $18(62.1)$ & $26(74.3)$ & $15(44.1)$ & $21(61.7)$ \\
\hline Left & $11(37.9)$ & $9(25.7)$ & $19(55.9)$ & $13(38.2)$ \\
\hline Stroke type & & & & \\
\hline Infarct & $25(86.2)$ & $30(85.7)$ & $24(72.7)$ & $27(79.4)$ \\
\hline Haemorrhage & $4(13.8)$ & $5(14.3)$ & $9(27.3)$ & $7(20.6)$ \\
\hline OCSP Classification & & & & \\
\hline LAC & $8(26.67)$ & $2(5.7)$ & $7(20.6)$ & $4(11.1)$ \\
\hline PAC & $6(20)$ & $15(42.9)$ & $10(29.4)$ & $10(27.8)$ \\
\hline POC & $2(6.7)$ & $1(2.9)$ & $2(5.9)$ & $2(5.6)$ \\
\hline TAC & $8(26.7)$ & $10(28.6)$ & $8(23.5)$ & $11(30.6)$ \\
\hline Uncertain & $6(20)$ & $7(20)$ & $7(20.6)$ & $9(25)$ \\
\hline MRS at admission & & & & \\
\hline $0-1$ & $21(80.8)$ & $22(64.7)$ & $22(66.7)$ & $19(54.28)$ \\
\hline $2-3$ & $3(11.5)$ & $7(20.6)$ & $6(18.2)$ & $14(40)$ \\
\hline $4-5$ & $2(7.6)$ & $8(23.5)$ & $5(15.15)$ & $2(5.71)$ \\
\hline Cog-4 & & & & \\
\hline Median (IQR) & $2(0,4)$ & $2(0,3)$ & $1(0,5)$ & $2(1,3)$ \\
\hline
\end{tabular}

NIHSS: National Institute of Health Stroke Scale; OCSP: Oxford Community Stroke Project; LAC: lacunar; PAC: partial anterior circulation; POC: posterior circulation; TAC: total anterior circulation. MRS: modified Rankin Scale.

\section{Patient activity patterns across the four sites}

The 27 observational activity codes were grouped into eight core categories. Figure 1 presents the proportion of observations per activity type for each site. Here, averages across sites are reported. Across the four sites, participants spent on average $37 \%$ of their time observed sleeping or lying undertaking no activity. Another $31 \%$ of their day was spent in low level physical activities such as sitting or watching TV. The mobility category referred to activities related to mobilization, positioning and practicing sitting/standing balance and accounted for $4 \%$ of observations across the four sites. On average, $14 \%$ of total observations were spent in the practice of self-care ADL activities (e.g. washing, dressing, grooming), either with help or independently, while motor ADL (e.g. transfers, walking, using wheelchair etc.) accounted for 3\% and extended ADL for $6 \%$ of observations across sites (included activities such as reading, writing, using a phone or computer and leisure activities). Psychological and patient and carer educational interventions were rarely observed $(0.8 \%)$ across the four sites.

Figure 1. Proportion of observations per type of activity across the four sites.

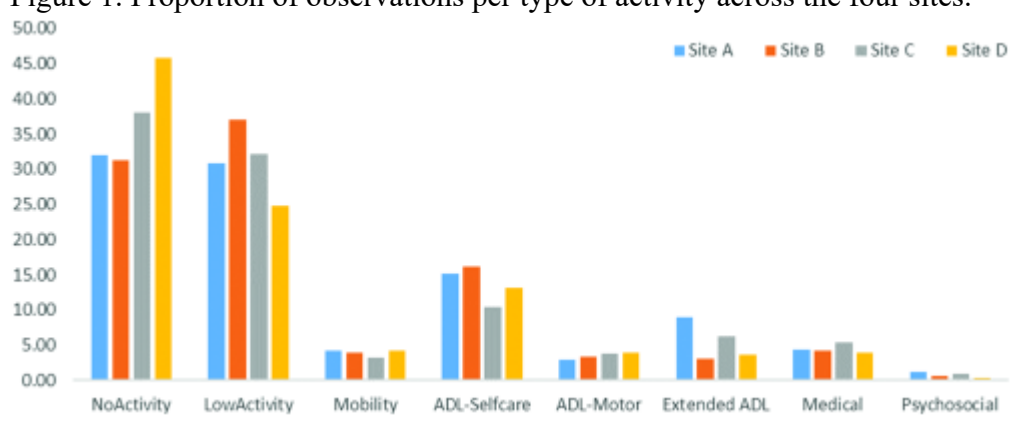

\section{Interactions with others}

Figure 2 shows the proportion of observations that patients spent on their own or interacting with someone else (categories not mutually exclusive). When reporting nursing data, nurses and care assistants were combined. Averaging across the four units, patients spent on average $60 \%$ of observations alone and $18 \%$ interacting with a healthcare 
professional, of which therapy (occupational therapy, physiotherapy, speech and language therapy) accounted for $10 \%$ of observations. More specifically across the four sites, participants spent on average $2.6 \%$ of their time in the presence of an occupational therapist and 3.7\% with a physiotherapist. Interactions with rehabilitation assistants was, on average, $2.7 \%$ of observations in three of the four sites where the role was in place. Time with doctors accounted for $1.2 \%$ of observations and nursing staff were present for $6.3 \%$ of observations. Across the units, patients' most frequent contact, at $19 \%$ of observations, was with family members. Similar patterns of findings were seen when analyses focused on the observations made between $8 \mathrm{am}$ and $5 \mathrm{pm}$ on working days.

Figure 2. Percentage of observations spent alone or in contact with others.

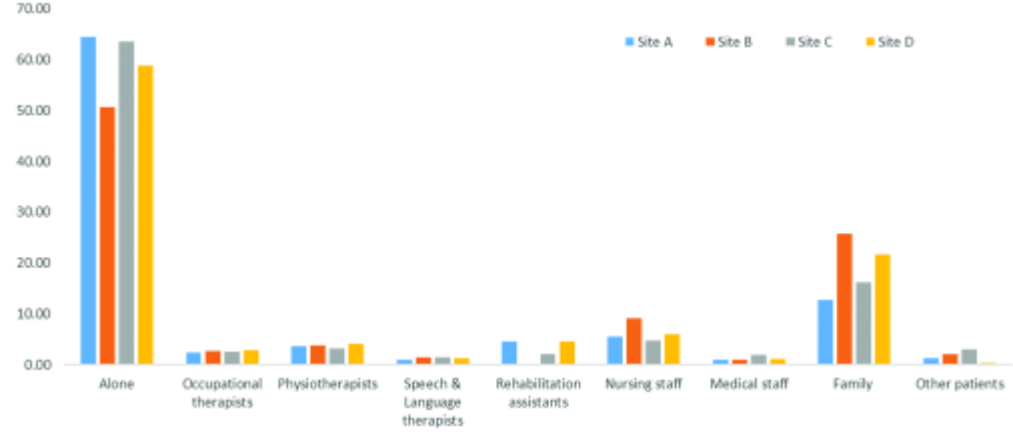

Patients' activity patterns when alone or interacting with someone can be seen in Figure 3. Across the four sites, when patients were alone they spent $57 \%$ of their time inactive. They were most physically active in the presence of therapists and the highest levels of self-care ADL practice, at $20 \%$ of observations, were in the presence of nursing staff.

Figure 3. Average proportion of observations in different activities per people present across the four sites.

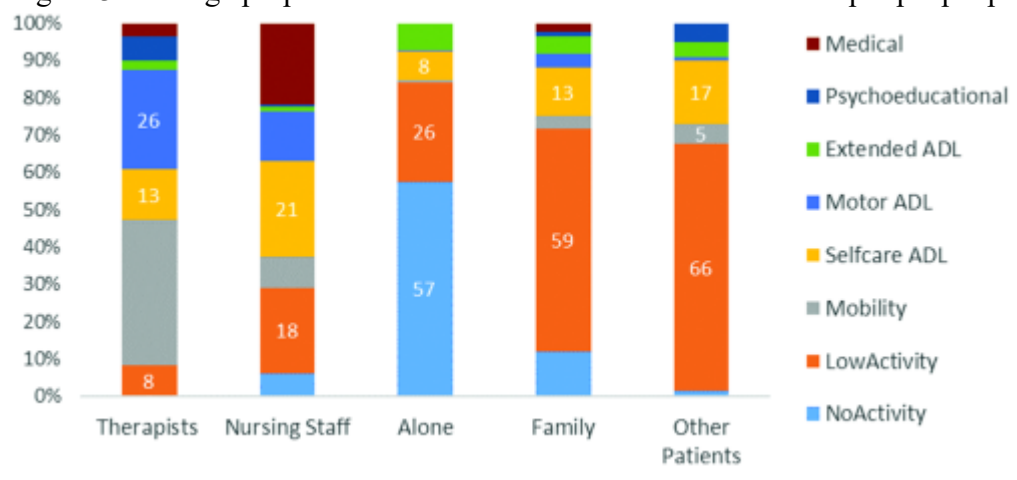

\section{Location of activity}

Participants across the four units spent $88 \%$ of the time they were observed in or by their beds (Figure 4). Activity in the gym accounted on average for $2 \%$ of observations. Interactions in a therapy kitchen and off-hospital activity occurred only in two sites.

Figure 4. Proportion of observations spent in different locations across the four sites. 


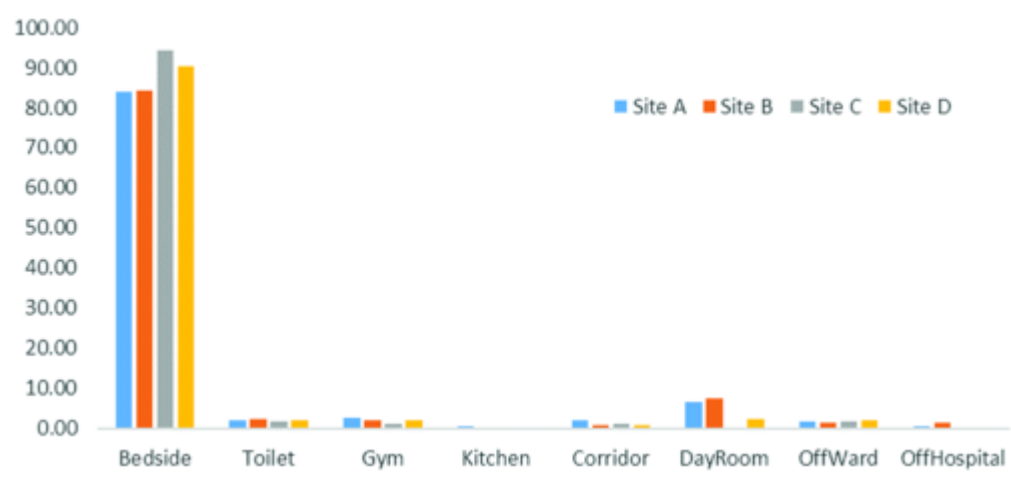

\section{Comparisons between units}

Significant differences were found between the sites in the proportion of time patients spent alone as well as the likelihood of them engaging in self-care ADL and extended ADL activities, after correcting for multiple testing (Table 3). The units did not differ significantly in the time patients spent with occupational therapists and physiotherapists however, significant differences were seen with regards to the presence of rehabilitation assistants (in the three units where the role was in place) and nursing staff. Differences in the proportion of time patients spent in the day room (Table 4) possibly reflect the lack of an accessible day room in site D during two of our five visits in the unit. No other significant differences in location were observed.

Table 3. Comparisons of activity and people observational categories across the four sites.

\begin{tabular}{|c|c|c|c|c|c|c|c|c|c|}
\hline \multicolumn{10}{|c|}{ Activity categories } \\
\hline & \multicolumn{3}{|c|}{ Low activity } & \multicolumn{3}{|c|}{ ADL self-care } & \multicolumn{3}{|c|}{ Extended ADL } \\
\hline & RRR & 2 $95 \% \mathrm{CI}$ & $p$ & RRR & $95 \% \mathrm{CI}$ & $p$ & RRR & $95 \% \mathrm{CI}$ & $p$ \\
\hline \multicolumn{2}{|c|}{ B vs A 1.36} & $0.92,2.02$ & 0.12 & 1.16 & $0.90,1.47$ & 0.24 & +0.34 & $0.17,0.80$ & $<0.05$ \\
\hline \multicolumn{2}{|c|}{ C vs A 0.96} & $0.65,1.41$ & 0.84 & 0.62 & $0.48,0.80$ & $<0.0001$ & 0.68 & $0.32,1.42$ & 0.31 \\
\hline \multicolumn{2}{|c|}{ D vs A 0.59} & $0.40,0.88$ & $<0.0001$ & 0.62 & $0.48,0.80$ & $<0.0001$ & 0.31 & $0.15,0.66$ & $<0.0001$ \\
\hline \multicolumn{2}{|c|}{ C vs B 0.70} & $0.48,1.03$ & 0.07 & 0.53 & $0.42,0.69$ & $<0.0001$ & 1.82 & $0.86,3.84$ & 0.11 \\
\hline \multicolumn{2}{|c|}{ D vs B 0.44} & $0.30,0.64$ & $<0.0001$ & 0.53 & $0.42,0.68$ & $<0.0001$ & 0.83 & $0.39,1.79$ & 0.64 \\
\hline \multicolumn{2}{|c|}{ D vs C 0.62} & $0.42,0.91$ & $<0.05$ & 1.00 & $0.78,1.29$ & 0.98 & 0.46 & $0.21,0.95$ & $<0.5$ \\
\hline \multicolumn{10}{|c|}{ People present } \\
\hline & \multicolumn{3}{|c|}{ Alone } & \multicolumn{3}{|c|}{ Rehabilitation assistants } & \multicolumn{3}{|c|}{ Nurses } \\
\hline & OR & $95 \% \mathrm{CI}$ & $p$ & OR & $95 \% \mathrm{CI}$ & $p$ & OR & $95 \% \mathrm{CI}$ & $p$ \\
\hline \multicolumn{2}{|c|}{ B vs A 0.48} & $0.33,0.71$ & $<0.0001$ & & NA & & 2.13 & $1.39,3.27$ & $<0.0001$ \\
\hline \multicolumn{2}{|c|}{ C vs A 0.93} & $0.63,1.37$ & 0.73 & 0.29 & $0.15,0.56$ & $<0.0001$ & 1.12 & $0.72,1.74$ & 0.61 \\
\hline \multicolumn{2}{|c|}{ D vs A 0.71} & $0.48,1.04$ & 0.08 & 1.02 & $0.58,1.80$ & 0.95 & 0.97 & $0.62,1.52$ & 0.89 \\
\hline \multicolumn{2}{|c|}{$\begin{array}{ll}\text { C vs B } 1.93 \\
\end{array}$} & $1.32,2.83$ & $<0.0001$ & & NA & & 0.52 & $0.34,0.80$ & $<0.05$ \\
\hline \multicolumn{2}{|c|}{ D vs B 1.47} & $1.0,2.15$ & $<0.05$ & & NA & & 0.45 & $0.30,0.70$ & $<0.0001$ \\
\hline \multirow[t]{3}{*}{ D vs $C$} & 0.76 & $0.52,1.11$ & 0.16 & 3.55 & $1.83,6.91$ & $<0.0001$ & 0.86 & $0.56,1.34$ & 0.52 \\
\hline & & althcare assi & istants & & Family & & & Other patie & \\
\hline & OR & $95 \% \mathrm{CI}$ & $p$ & OR & $95 \% \mathrm{CI}$ & $p$ & OR & $95 \% \mathrm{CI}$ & $p$ \\
\hline \multicolumn{2}{|c|}{ B vs A 1.08} & $0.66,1.78$ & 0.75 & 3.47 & $1.76,6.81$ & $<0.0001$ & 1.76 & $0.33,9.42$ & 0.51 \\
\hline \multicolumn{2}{|c|}{ C vs A 0.57} & $0.34,0.95$ & $<0.05$ & 1.39 & $0.71,2.74$ & 0.34 & 0.95 & $0.16,5.47$ & 0.96 \\
\hline \multicolumn{2}{|c|}{ D vs A 1.07} & $0.65,1.75$ & 0.79 & 2.47 & $1.26,4.84$ & $<0.0001$ & 0.15 & $0.02,1.34$ & 0.09 \\
\hline \multicolumn{2}{|c|}{ C vs B 0.53} & $0.32,0.87$ & $<0.05$ & 0.40 & $0.21,0.77$ & $<0.0001$ & 0.54 & $0.10,2.86$ & 0.47 \\
\hline
\end{tabular}




Activity categories
\begin{tabular}{|c|c|c|c|c|c|c|c|c|c|}
\hline & \multicolumn{3}{|c|}{ Low activity } & \multicolumn{3}{c|}{ ADL self-care } & \multicolumn{3}{c|}{ Extended ADL } \\
\hline RRR & $95 \%$ CI & $p$ & RRR & $95 \%$ CI & $p$ & RRR & $95 \%$ CI & \multicolumn{1}{c|}{$p$} \\
\hline D vs B & 0.98 & $0.60,1.60$ & 0.95 & 0.71 & $0.37,1.36$ & 0.31 & 0.09 & $0.01,0.72$ & $<0.05$ \\
\hline D vs C & 1.87 & $1.13,3.09$ & $<0.05$ & 1.77 & $0.92,3.41$ & 0.09 & 0.16 & $0.02,1.38$ & 0.09 \\
\hline
\end{tabular}

Only categories where significant differences were found are presented in the table; RRR: relative risk ratio; OR: odds ratio; $p$ : unadjusted $p$-value.

Table 4. Comparisons for "Day room" category across the four sites.

\begin{tabular}{|c|c|c|c|c|}
\hline & \multicolumn{4}{|c|}{$\begin{array}{l}\text { Location } \\
\text { Day room }\end{array}$} \\
\hline & RRR & $95 \% \mathrm{CI}$ & & $p$ \\
\hline $\mathrm{B}$ vs $\mathrm{A}$ & 1.73 & $0.49,4.81=$ & $\bar{E}$ & 0.29 \\
\hline $\mathrm{C}$ vs $\mathrm{A}$ & 350.351 & $19.86,1406.2$ & & 0001 \\
\hline D vs A & 0.004 & $0,0.21$ & & 0001 \\
\hline C vs B & 657.676 & $65.10,2367.50$ & & 0001 \\
\hline D vs B & 0.25 & $0,0.10$ & & 0001 \\
\hline D vs $C$ & 15.03 & $11.04,92.58$ & & 0.05 \\
\hline
\end{tabular}

RRR: relative risk ratio; $p$ : unadjusted $p$ value.

\section{Discussion}

This study examined how key features of evidence based stroke rehabilitation are delivered in clinical practice as reflected in how patients' time was spent. Findings suggested that patients spent a big proportion of their time in the stroke rehabilitation units inactive (37\%) and alone $(60 \%)$. Activity away from the bedside was scarce $(12 \%)$ while rehabilitation in the gym was rarely observed (2\%). Of particular interest was the practice of ADL tasks; results suggested that patients spent a small proportion of their time engaging in self-care ADL (14\%), either with help or independent, and had limited opportunities for the practice of motor ADL (3\%). Significant variation was observed among the participating rehabilitation units on a number of components of rehabilitation practice but not in the amount of time interacting with occupational therapy and physiotherapy staff members.

Results are consistent with those reported from early studies [10] in the United Kingdom and abroad although variation in sample characteristics and classification of activity categories impede direct comparisons. The proportion of therapy time (10\%) was slightly higher than previously reported in England [16,26], however, opportunities for physical activity outside formal therapy remained limited. The prolonged inactivity observed in this study raises questions about whether patients have adequate opportunities to practice tasks necessary to maintain the skills gained in therapy and promote independence.

This was the first study to examine participants' levels of engagement specifically in ADL practice. Previous mapping studies classified certain ADL and extended ADL activities as "non-therapeutic" or "low level physical activity" obscuring their role in rehabilitation $[16,27,28]$. In the light of research evidence linking intensive ADL practice with better functional outcomes [1], our findings highlight the need to enhance this rehabilitation component and maximise opportunities for patient involvement in therapeutic ADL.

All but one units (unit B) met the number of therapy staff members recommended for the operation of an evidence-based stroke unit [2]. This suggests that the low activity levels may not be solely attributed to staffing levels but differences in other organisational processes. In line with this hypothesis, a recent study by Clarke et al. [29] identified organisational factors, such as the time spent in information exchange, as influencing the intensity of thera- 
py provision. In this study, the highest levels of therapeutic input were seen in units where a strong presence of rehabilitation assistants was also observed. In units where patients were most active and less lonely, patients spent more time interacting with nursing staff, family members and other patients and made frequent use of the day room. These observations do not imply causality between these factors but could be used for hypothesis generation in future studies.

When interpreting the findings, we need to be mindful of limitations of the study design. As we did not observe patients behind closed curtains we needed to rely on accurate reports from staff members on the type of patient activity. The possible effects of the observers' presence should also be taken into consideration. Reactivity effects are inherent in observational methodology [30] although the amount of time observers spent in the units may have promoted participant habituation to their presence and normalisation of behaviour [31]. Behavioural mapping also suffers from an inability to capture the contextual elements of interactions. The wider REVIHR study also included a realist evaluation to uncover explanatory mechanisms underpinning the implementation of evidence-based rehabilitation (parallel publication under review elsewhere).

In England, improvement efforts to date have mainly focused in achieving $45 \mathrm{~min}$ of therapy per day. Experimental and clinical studies highlight the importance of a stimulating social, cognitive and physical environment in maximising stroke survivors' potential for recovery $[32, \bar{O}$ Our findings showed limited opportunities for interaction

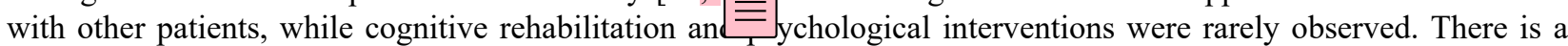
need to look beyond the 45-min target and towards enhancing patient experience outside formal therapy time. Future studies should evaluate pragmatic approaches to promote a stimulating and rehabilitation conducive environment within existing resources.

Behavioural mapping allowed us to identify potential targets for future quality improvement efforts. To date, attempts to increase stroke survivor activity levels have mainly targeted the practice of occupational and physiotherapy staff members. Our findings point to untapped opportunities for more involvement of the family and the wider multidisciplinary team. While interventions to promote carers' participation in stroke rehabilitation have received increasing attention in the literature, differences between their scope, context and mode of delivery make it hard to conclude on their value. Some gains have been reported in muscle strength [35] and the use of the affected limb [36], but controlled studies have failed to find an effect on patients' mobility and functional status [37,38]. At the time of our observations some attempts to give families a more active role in the rehabilitation process were reported by the teams, but these were isolated efforts which lacked a systematic approach. It is, therefore, unclear whether the observed differences in the proportion of time patients spent with family reflect these initiatives or variations in visiting hours. Clinical guidelines recognise family involvement in rehabilitation as a component of evidence-based practice [2]; as such, there is a need to develop a framework to guide and formalise clinicians' initiatives through the evaluation of strategies realistic and feasible to implement in clinical practice.

The participating units differed significantly in the proportion of time patients spent with rehabilitation assistants but not in the time spent with registered therapists. Introducing or further developing the role of rehabilitation assistants may also serve to enhance the intensity of rehabilitation interventions and release time for therapists to focus on more complex cases, increasing the efficiency of ward activities. This is especially important within the current economic climate as there is a need to increase patients' activity without increasing therapist staffing levels.

Our observations suggested that it was mostly in the presence of nursing staff that patients engaged in the practice of self-care ADL. This finding demonstrates the potential for nursing staff to maximise opportunities for practice of functional tasks and support patients' efforts to maintain their dignity and regain their independence. Earlier studies have provided some evidence of the benefits of nursing staff's involvement in promoting mobilisation [27] and encouraging autonomous exercise [39,40]. Future research should evaluate pragmatic approaches to encourage collaborative synergy between nursing and therapy professions, enhance communication and promote a shared rehabilitation ethos across the multidisciplinary team.

Overall, our findings suggest that despite the strong research and policy drivers over the past 10 years in England, stroke survivors continue to spend most of their time inactive and alone. This emphasises the need for quality improvement efforts which will optimise the capacity of rehabilitation units to provide a rehabilitation focused environment necessary to support patients in regaining their skills and maintaining independence during a critical for their recovery period. 


\section{Acknowledgments}

We wish to acknowledge Prof P. Langhorne and Prof T. Robinson for offering expert advice throughout the study. We are grateful to the stroke survivors and staff members in the four participating stroke rehabilitation units for their cooperation and engagement.

\section{Disclosure statement}

The authors report no conflicts of interest.

\section{References}

1. Stroke Unit Trialists' Collaboration. Organised inpatient (stroke unit) care for stroke. Cochrane Database Syst Rev. 2013;9:CD000197.

2. Intercollegiate Stroke Working Party. National clinical guideline for stroke. 5th ed. London: Royal College of Physicians. 2016. Available from: https://www.strokeaudit.org/SupportFiles/Documents/Guidelines/2016-NationalClinical-Guideline-for-Stroke-5t-(1).aspx

3. Hsieh C, Huang H, Philbert D, et al. Effect of rehabilitation intensity on mortality risk after stroke. Arch Phys Med Rehabil. 2018;99(6):1042-1048.

4. Langhorne P, Bernhardt J, Kwakkel G. Stroke rehabilitation. Lancet. 2011;377(9778):1693-1702.

5. Kwakkel G, van Peppen R, Wagenaar RC, et al. Effects of augmented exercise therapy time after stroke: a metaanalysis. Stroke. 2004;35(11):2529-2539.

6. Wade DT. Rehabilitation-a new approach. Part four: a new paradigm, and its implications. Clin Rehabil. 2016;30(2):109-118.

7. Hebert D, Lindsay MP, McIntyre A, et al. Canadian stroke best practice recommendations: stroke rehabilitation practice guidelines. Int J Stroke. 2016;11(4):459-484.

8. Stroke Foundation. Clinical guidelines for stroke management. Melbourne Australia, 2017. Available from: https://informme.org.au/guidelines.

9. Scottish Intercollegiate Guidelines Network. Management of patients with stroke or TIA: Assessment, investigation, immediate management and secondary prevention. A national clinical guideline. 2008. Available from: http:// www.sign.ac.uk/sign-108-management-of-patients-with-stroke-or-tia-assessment-investigation-immediate-management-a.html.

10. West T, Bernhardt J. Physical activity patterns of acute stroke patients managed in a rehabilitation focused stroke unit. BioMed Res Int. 2013;2013:438679.

11. Fini NA, Holland AE, Keating J, et al. How physically active are people following stroke? Systematic review and quantitative synthesis. Phys Ther. 2017;97(7):707-717.

12. Royal College of Physicians Intercollegiate Stroke Working Party. Sentinel Stroke National Audit Programme (SSNAP). Clinical audit; 2015. Available from: https://www.strokeaudit.org/results.aspx.

13. Royal College of Physicians Intercollegiate Stroke Working Party. Sentinel Stroke National Audit Programme (SSNAP). Acute organisational audit; 2015. Available from: https://www.strokeaudit.org/results.aspx.

14. Walker MF, Fisher RJ, Korner-Bitensky N, et al. From what we know to what we do: translating stroke rehabilitation research into practice. Int J Stroke. 2013;8(1):11-17.

15. Cadilhac DA, Fisher R, Bernhardt J. Online supplement: how to do health services research in stroke: a focus on performance measurement and quality improvement. Int J Stroke. 2018;31(2):166-174.

16. De Wit L, Putman K, Dejaeger E, et al. Use of time by stroke patients: a comparison of four european rehabilitation centers. Stroke. 2005;36(9):1977-1983.

17. Legg LA, Drummond A, Leonardi-Bee J, et al. Occupational therapy for patients with problems in personal activities of daily living after stroke: a systematic review of randomised trials. BMJ. 2007;335(7626):922. 
18. WHO (World Health Organization). Cerebrovascular disease: a clinical and research classification. Geneva: WHO; 1978.

19. Mahoney FI, Barthel DW. Functional evaluation: the Barthel index. Md State Med J. 1965;14:61-65.

20. Bamford J, Sandercock P, Dennis M, et al. Classification and natural history of clinically identifiable subtypes of cerebral infarction. Lancet. 1991;337(8756):1521-1526.

21. Brott T, Adams HP, Olinger CP, et al. Measurements of acute cerebral infarction: a clinical examination scale. Stroke. 1989;20(7):864-870.

22. Swieten JC, van Koudstaal PJ, Visser MC, et al. Interobserver agreement for the assessment of handicap in stroke patients. Stroke. 1988;19(5):604-607.

23. Cumming TB, Blomstrand C, Bernhardt J, et al. The NIH stroke scale can establish cognitive function after stroke. Cerebrovasc Dis. 2010;30(1):7-14.

24. Goldstein H. Multilevel statistical models. 4th ed. UK $\equiv$ niley \& Sons Ltd; 2011.[AQ3]

25. Leckie G, Charlton C. Runmlwin: a program to run the IVLwiN multilevel modeling software from within. J Stat Soft. 2013;52(11):1-40.

26. Wellwood I, Langhorne P, McKevitt C, et al. An observational study of acute stroke care in four countries: the European registers of stroke study. Cerebrovasc Dis. 2009;28(2):171-176.

27. Bernhardt J, Chitravas N, Meslo IL, et al. Not all stroke units are the same. A comparison of physical activity patterns in Melbourne, Australia, and Trondheim, Norway. Stroke. 2008;39(7):2059-2065.

28. Skarin M, Sjöholm A, Nilsson ÅL, et al. A mapping study on physical activity in stroke rehabilitation: establishing the baseline. J Rehabil Med. 2013;45(10):997-1003.

29. Clarke DJ, Burton LJ, Tyson SF, et al. Why do stroke survivors not receive recommended amounts of active therapy? Findings from the ReAcT study, a mixed-methods case-study evaluation in eight stroke units. Clin Rehabil. 2018;32(8):1119-1132.

30. Robson C. Real world research. 2nd ed. UK $\equiv$ ckwell Publishing; 2002.[AQ4]

31. Kerlinger FN, Lee HB. Foundations of behavioural research. 4th ed. UK $\equiv$ rcourt College Publishers; 2000. [AQ5]

32. Craft TKS, Glasper ER, McCullough L, et al. Social interaction improves experimental stroke outcome. Stroke. 2005;36(9):2006-2011.

33. Janssen H, Bernhardt J, Collier JM, et al. An enriched environment improves sensorimotor function post-ischemic stroke. Neurorehabil Neural Repair. 2010;24(9):802-813.[AQ6]

34. Särkämö T, Ripollés P, Vepsäläinen H, et al. Structural changes induced by daily music listening in the recovering brain after middle cerebral artery stroke: a voxel-based morphometry study. Front Hum Neurosci. 2014;8:245.

35. Maeshima S, Ueyoshi A, Osawa A, et al. Mobility and muscle strength contralateral to hemiplegia from stroke: benefit from self-training with family Support. Am J Phys Med Rehabil. 2003;82(6):456.

36. Harris JE, Eng JJ, Miller WC, et al. The role of caregiver involvement in upper-limb treatment in individuals with subacute stroke. Phys Ther. 2010;90(9):1302-1310.

37. Forster A, Dickerson J, Young J, et al. A structured training programme for caregivers of inpatients after stroke (TRACS): a cluster randomised controlled trial and cost-effectiveness analysis. Lancet. 2013;382(9910):2069-2076.

38. Lindley RI, Anderson CS, Billot L, et al. Family-led rehabilitation after stroke in India (ATTEND): a randomised controlled trial. Lancet. 2017;390(10094):588-599.

39. Huijben-Schoenmakers M, Rademaker A, Scherder E, et al. Can practice undertaken by patients be increased simply through implementing agreed national guidelines?” An observational study. Clin Rehabil. 2013;27(6):513520 . 
40. Rensink M, Schuurmans M, Lindeman E, et al. Task-oriented training in rehabilitation after stroke: systematic review. J Adv Nurs. 2009;65(4):737-754.

\section{AUTHOR QUERIES}

Query: AQ0: Please review the table of contributors below and confirm that the first and last names are structured correctly and that the authors are listed in the correct order of contribution. This check is to ensure that your names will appear correctly online and when the article is indexed.

\begin{tabular}{|c|c|c|c|}
\hline Sequence & Prefix Given name(s) & Surname & Suffix \\
\hline 1 & Niki & Chouliara & \\
\hline 2 & Rebecca & Fisher & \\
\hline 3 & Brian & Crosbie & \\
\hline 4 & Boliang & Guo & \\
\hline 5 & Nikola & Sprigg & \\
\hline 6 & Marion & Walker & \\
\hline
\end{tabular}

\section{Response: Ok}

Query: AQ1: Please confirm the author names as set in the proof are accurate. Response: Ok

Query: AQ2: Please check "Funding: section has been typeset correctly and correct if this is inaccurate.

Response: This research was funded by the National Institute for Health Research (NIHR) as part of the Collaboration for Leadership in Applied Health Research and Care East Midlands (CLAHRC EM). The views expressed are those of the authors and not necessarily those of the NIHR or the Department of Health and Social Care.

Query: AQ3: Please provide missing publisher city for the "24" references list entry.

Response: Answered within text

Query: AQ4: Please provide missing publisher city for the " 30 " references list entry.

Response: Answered within text

Query: AQ5: Please provide missing publisher city for the " 31 " references list entry.

Response: Answered within text

Query: AQ6: The reference " 33 " is listed in the references list but is not cited in the text. Please either cite the reference or remove it from the references list.

Response: Answered within text

Query: AQ7: The ORCID details of the authors have been validated against ORCID registry. please check the ORCID ID details of the authors.

Response: Please add ORCID details for Rebecca Fisher 0000-0001-6866-6341Marion Walker 0000-0002-3534-591X

\section{COMMENTS}

C1 Author: Since first submitting the article our Division name has changed to:Division of Rehabilitation, Ageing and Wellbeing;

C2 Author: Division of Rehabilitation, Ageing and Wellbeing;

C3 Author: reference 33 to be added here so it reads [32,33,34];

C4 Author: Chichester;

C5 Author: Oxford;

C6 Author: UK to be deletedForth Worth, USA; 
C7 Author: Please can A, B, C and D in this first row be centrered to match the rest of the column?; C8 Author: Please can this column be formatted the same way as other columns (i.e. to the left); C9 Author: Please can Location and Day room be centered across the 3 columns below.; 